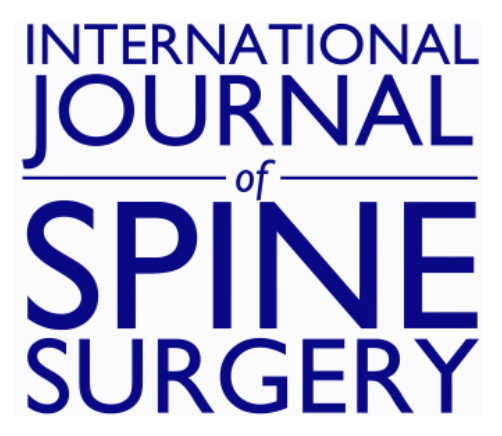

\title{
Surgical Treatment for Discogenic Low-Back Pain: Lumbar Arthroplasty Results in Superior Pain Reduction and Disability Level Improvement Compared With Lumbar Fusion
}

Fred H. Geisler

Int J Spine Surg 2007, 1 (1) 12-19

doi: https://doi.org/10.1016/SASJ-2006-0002-RR

http://ijssurgery.com/content/1/1/12

This information is current as of April 26, 2023.

Email Alerts Receive free email-alerts when new articles cite this article. Sign up at:

http://ijssurgery.com/alerts

The International Journal of Spine Surgery

2397 Waterbury Circle, Suite 1,

Aurora, IL 60504, Phone: +1-630-375-1432 


\section{Surgical Treatment for Discogenic Low-Back Pain: Lumbar Arthroplasty Results in Superior Pain Reduction and Disability Level Improvement Compared With Lumbar Fusion}

Fred H. Geisler, MD, PhD

\section{ABSTRACT}

\section{Background}

The US Food and Drug Administration approved the Charité artificial disc on October 26, 2004. This approval was based on an extensive analysis and review process; 20 years of disc usage worldwide; and the results of a prospective, randomized, controlled clinical trial that compared lumbar artificial disc replacement to fusion. The results of the investigational device exemption (IDE) study led to a conclusion that clinical outcomes following lumbar arthroplasty were at least as good as outcomes from fusion.

\section{Methods}

The author performed a new analysis of the Visual Analog Scale pain scores and the Oswestry Disability Index scores from the Charité artificial disc IDE study and used a nonparametric statistical test, because observed data distributions were not normal. The analysis included all of the enrolled subjects in both the nonrandomized and randomized phases of the study.

\section{Results}

Subjects from both the treatment and control groups improved from the baseline situation $(P<.001)$ at all follow-up times $(6$ weeks to 24 months). Additionally, these pain and disability levels with artificial disc replacement were superior $(P<.05)$ to the fusion treatment at all follow-up times including 2 years.

\section{Conclusions}

The a priori statistical plan for an IDE study may not adequately address the final distribution of the data. Therefore, statistical analyses more appropriate to the distribution may be necessary to develop meaningful statistical conclusions from the study. A nonparametric statistical analysis of the Charité artificial disc IDE outcomes scores demonstrates superiority for lumbar arthroplasty versus fusion at all follow-up time points to 24 months.

Key Words lumbar arthroplasty, total disc replacement, clinical trial, statistical analysis. SAS Journal. Winter 2007; 1; 12-19. DOI: SASJ-2006-0002-RR

\section{INTRODUCTION}

Low-back pain is the second most common reason (after the common cold) for visits to primary-care physicians. ${ }^{1} \mathrm{Up}$ to $80 \%$ of individuals in the United States will experience low-back pain at some point in their lives. ${ }^{2}$ Approximately 5\% of this group will progress to a condition of chronic back pain, much of which is attributable to degenerative disc disease (DDD), the leading cause of pain and disability in the United States. ${ }^{3,4}$ The exact incidence and prevalence of DDD is unknown, because many cases are asymptomatic and therefore do not trigger physician visits. $^{5}$

Examples of non-operative care for chronic low-back pain include allowing time for a natural healing mechanism to work, physical therapy, exercise, stretching, epidural steroid injections, and chiropractic care. Approximately 870,000 patients develop degenerative disc disease and a posteriorly directed dislodged disc fragment that occurs with compression of the exiting or transversed nerve root at the disc level, thereby producing sciatic pain and mechanical traction clinical signs. ${ }^{6,7}$ If these patients do not respond to nonsurgical care, then a discectomy or a microdiscectomy may be necessary for pain relief.

A fusion procedure is the most common surgical treatment for DDD of the lumbar spine. More than 200,000 lumbar fusion procedures are performed in the United States each year, though not all of them to treat DDD. ${ }^{8}$ After surgery, mature, healed fusion bone may take from 6 months to 2 years to develop and 
rehabilitation of the patient may require a similar time to be completed to achieve the maximum successful clinical benefit of the procedure.

Though lumbar artificial disc technology has been used commercially in Europe since 1987, the first disc implanted in the United States at the Texas Back Institute (Plano, Tex) by Scott Blumenthal was in March 2000 at the start of the US Food and Drug Administration (FDA) investigational device exemption (IDE) trial. Therefore, lumbar arthroplasty is a relatively new procedure to treat lumbar DDD in the United States. The hypothesized benefits of arthroplasty over fusion procedures with significant clinical benefit include (1) reduction or elimination of disc-derived (also known as discogenic) pain; (2) restoration and maintenance of normal segmental range of motion and sagittal balance; and (3) potential reduction or retardation of progressive adjacent-level DDD, which necessitates further surgical intervention involving not only reducing the forces and angulation of the adjacent level compared with a fusion but also normalizing the adjacent level biomechanics. ${ }^{9}$

On October 26, 2004, the FDA approved the world's first lumbar artificial disc, the Charité artificial disc (DePuy Spine, Raynham, Mass), for use in the United States. ${ }^{10}$ In doing so, the FDA followed the recommendation of its expert Orthopaedic and Rehabilitation Devices Panel, which on June 2, 2004, unanimously recommended approval. ${ }^{11}$ The FDA decision was based on the results of a prospective, randomized, controlled clinical trial that compared lumbar artificial disc replacement to anterior lumbar interbody fusion (ALIF) and on 20 years of worldwide surgical experience. FDA approval meant that the manufacturer could market the device as safe and effective for the treatment of single-level lumbar DDD in indicated patients at either the L4-5 or L5-S1 level.

This clinical trial was the first in the history of spine surgery to compare two different surgical treatments for lumbar DDD according to a multicenter, prospective, randomized, controlled study design. The results of the study were published in peerreviewed journals, including Journal of Neurosurgery in September $2004^{9}$ and Spine in July 2005. ${ }^{12,13}$ Because of the prospectively specified non-inferiority design of the study and the complex FDA-required success/failure criteria, the primary conclusion of the study for FDA labeling purposes was that treatment with artificial disc replacement was clinically at least as good as a fusion procedure.

I present here new level I medical evidence that surgical treatment with single-level arthroplasty is not only "at least as good as" a fusion procedure, as the FDA label states, but that reduction in pain and disability improvement are highly statistically superior in patients receiving treatment with lumbar arthroplasty compared to both baseline and an anterior fusion procedure. A more rapid decrease in pain and disability (postoperative healing/recovery) was also noted in the Charité group compared to the fusion group.

\section{MATERIALS AND METHODS}

A multicenter, prospective, randomized, controlled trial was performed under an FDA-approved protocol (IDE\# G990303). Local institutional review board approval was obtained at all 14 study sites, and all subjects enrolled in the study gave written informed consent. The trial incorporated a non-inferiority design with a 2:1 randomization: treatment with artificial disc replacement versus the control, a fusion procedure. Enrollment in the study constituted 71 subjects in an initial nonrandomized treatment phase (approximately 5 subjects per site) and then a total of 304 subjects in the randomized phase: 205 in the treatment group and 99 in the control group. Subjects in the control group underwent an ALIF procedure with BAK threaded fusion cages (Zimmer Spine, Minneapolis, Minn) and bone graft. Subjects were assessed clinically and radiographically before surgery, 6 weeks after surgery, and then at 3, 6, 12, and 24 months after surgery. Demographics, inclusion/exclusion criteria, subject accountability, clinical outcomes, and all other detailed study information conforming to the CONSORT checklist were previously described by Blumenthal et al. ${ }^{12}$

The Oswestry Disability Index (ODI) is a 10-question validated measure (score 0-100) of disability and pain among the population with low-back pain. ${ }^{14}$ Subjects were required to complete an ODI questionnaire and a 0-100 Visual Analog Scale (VAS) pain questionnaire preoperatively and at each follow-up visit.

\section{STATISTICAL METHODS}

The statistical analysis of the ODI and VAS scores performed for the FDA and reported by Blumenthal et al., required the use of the Student's $t$ test. This methodology was prespecified in the statistical plan of the protocol (1) before FDA approval of the protocol, (2) before subsequent subject enrollment, and (3) before the results/distributions of the data were known. In the FDA-approved protocol the methodology could not be altered post hoc for FDA labeling claims. Using Student's $t$ test, mean ODI and VAS scores were significantly better in the treatment group compared with the control group at all followup time points except for the 2-year follow-up. ${ }^{12}$ These results, combined with the non-inferiority study design, resulted in the primary conclusion of the study: that treatment with artificial disc replacement is at least as good as a fusion procedure in properly indicated patients.

However, Student's $t$ test, by definition, assumes a normal distribution of data. Therefore, Student's $t$ test is simply not the appropriate test with which to analyze non-normally distributed data. The ODI and VAS scores reasonably approximated a 
normal distribution at baseline. At the 2-year follow-upthe endpoint of the study-the distributions were heavily nonsymmetric, skewed, and clearly not normally distributed (Figure 1). Using Student's $t$ test might be compared to driving a car on tires designed to be inflated to $35 \mathrm{psi}$, but only inflating them to $10 \mathrm{psi}$.

A more appropriate statistical test for analysis of nonnormally distributed data would be a nonparametric test such as the Wilcoxon rank sum test, which is appropriate for a non-normal data distribution. I performed a separate analysis of the ODI and VAS scores using the Wilcoxon rank sum test in which all subjects enrolled in both the nonrandomized and randomized phases of the treatment group $(n=276)$ were compared to the study control group (the complete FDA IDE dataset). The data utilized for this analysis were the same data submitted to the FDA as part of the postmarketing application submission - this was not a subset analysis. The results were verified by an independent third party (Stat Tech Services, Chapel Hill, NC) using the SAS version 8.2 statistical software package (SAS Institute, Cary, NC).

Figure 1
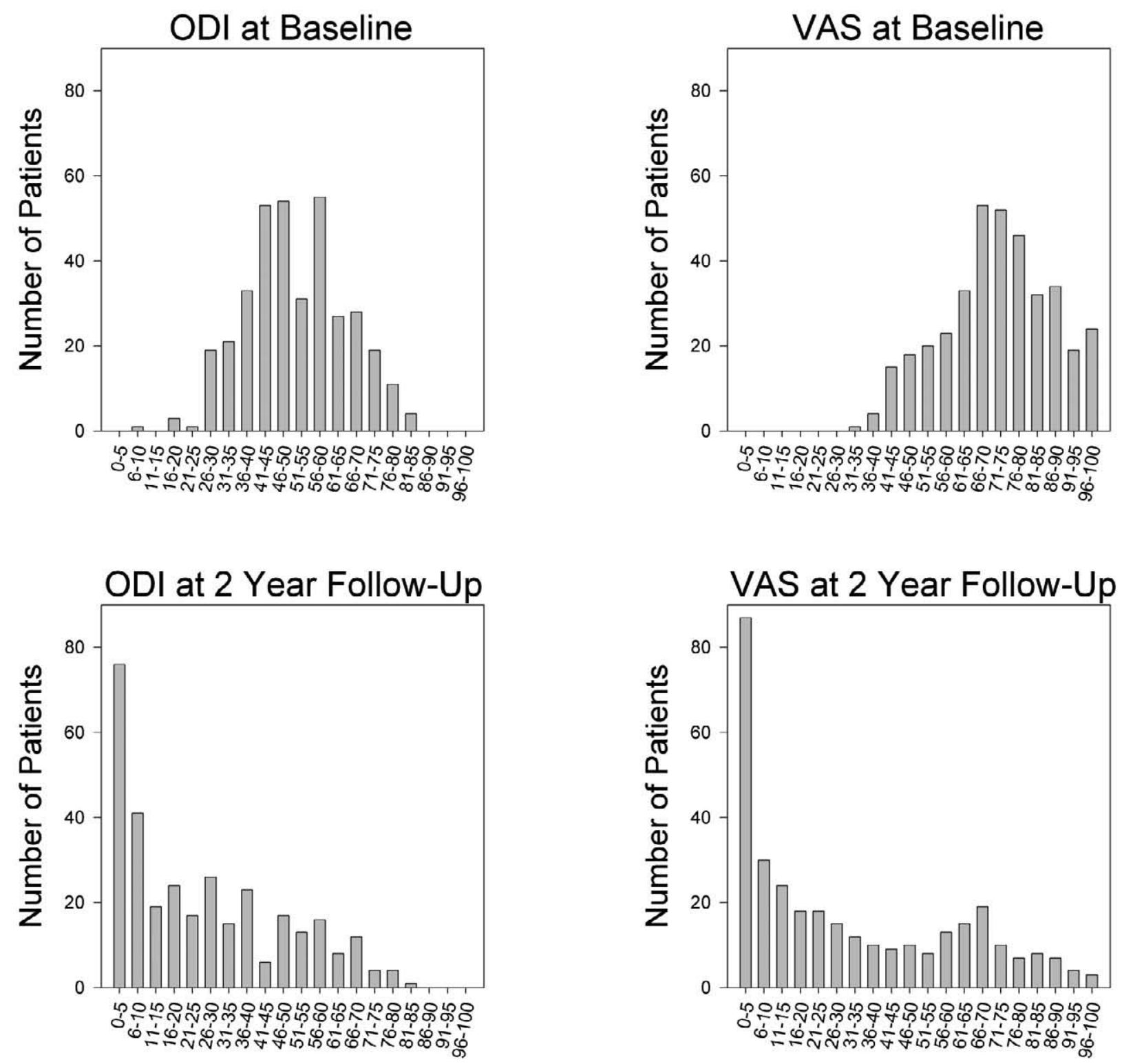

Distribution of VAS pain scores and ODI disability scores for all subjects (i.e., both treatment and control groups). A reasonable approximation of a normal distribution of scores is evident at baseline. However, at 2-year follow-up the distributions are not normal but skew towards the low cut-off end of both scales, indicating a favorable clinical response. 


\section{RESULTS}

Both the ODI and VAS scores had almost identical initial mean values for each of the 2 groups (treatment and control). Thus, the randomization worked in this study and no baseline corrections were necessary or used in the analysis of this data.

The nonparametric analysis of ODI and VAS scores demonstrated that subjects enrolled in both the treatment and the control groups had highly significantly lower scores at all time points compared to baseline, including the 2-year followup $(P<.001)$. The improved scores in both groups were $(1)$ sustained over the 2-year period, (2) monotonically decreasing, and (3) more than twice the difference considered to be of minimum clinical significance. ${ }^{15}$ This triad makes a placebo effect of surgery an unlikely explanation for the observed improvement. Furthermore, inspection of the recovery curves reveals significant improvement at 6 weeks with maintenance out to 2 years (Figures 2, 3).

Patients in the treatment group attained a greater proportion of the total 2-year recovery in this early phase of the postoperative period in both clinical indexes. Additionally, significantly lower scores occurred in the Charite artificial disc group compared to the control fusion group at all postoperative time points, including the 2 -year follow-up $(P<.05)$. These results demonstrate superiority of arthroplasty over fusion in indicated patients, according to these key clinical measures, and a major improvement from baseline in both treatment groups. The control group scores closely followed the results of the BAK cage IDE study described by Kuslich et al. ${ }^{16}$

\section{DISCUSSION}

Given the distribution of the ODI and VAS scores at 2 years as shown in Figure 1, it is clear that a nonparametric test is the appropriate statistical test for analyzing the ODI and VAS data from the Charité artificial disc clinical trial. This new analysis does not and cannot change the primary FDA study conclusion: that arthroplasty is "at least as good as" a fusion procedure. This limitation of labeling claims occurred because the FDA study was conducted with a non-inferiority design with prespecified criteria for clinical success. Furthermore, FDA claims of clinical superiority cannot emanate from non-inferiority studies that are not a priori sufficiently powered to demonstrate superiority of one treatment over another.

However, improvement in ODI score was a primary clinical endpoint of this study and improvement in VAS score was a secondary clinical endpoint. These 2 clinical outcome measures are the most relied-upon measures of clinical outcome following low-back surgery. There is no doubt, following this analysis, that subjects receiving arthroplasty attained superiority in improved pain and disability levels compared with baseline levels and at

Figure 2
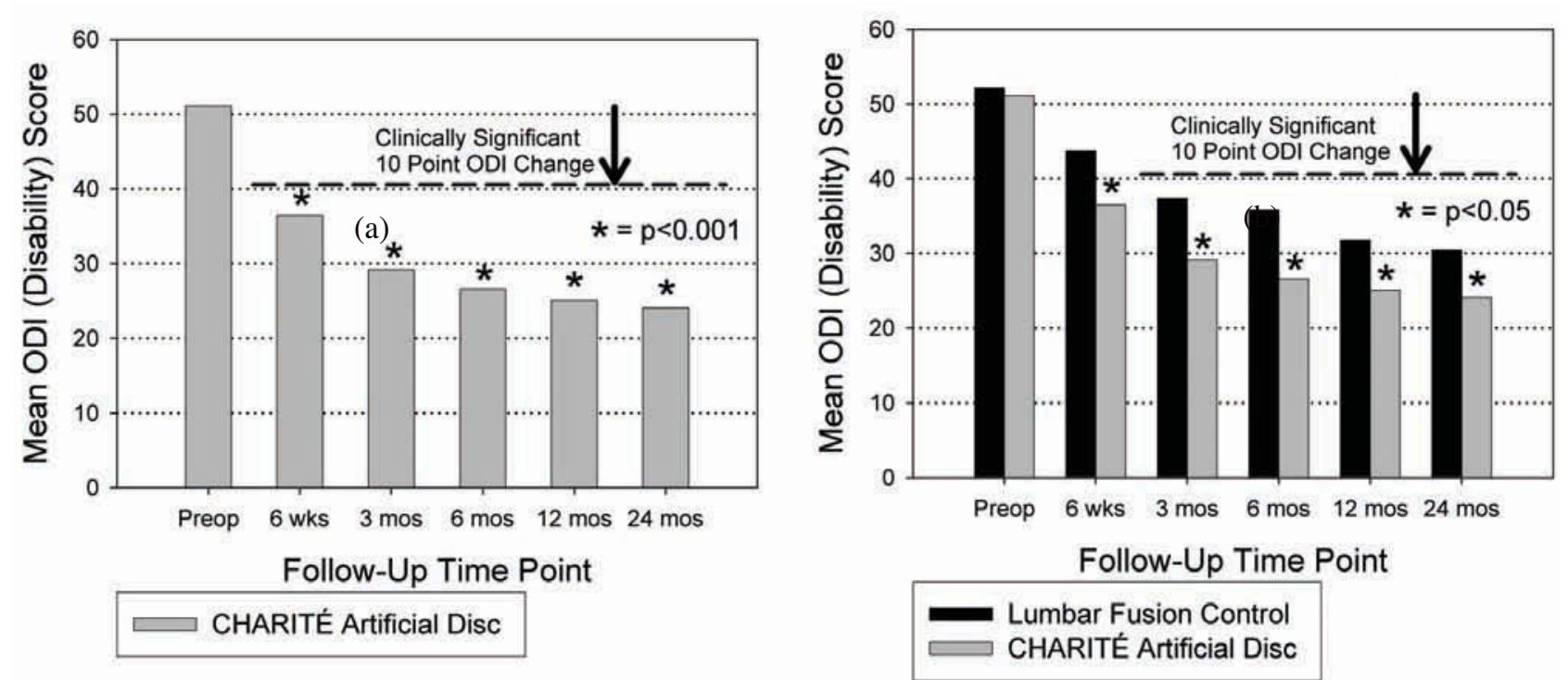

Mean ODI disability scores at baseline and at each protocol-specified follow-up time point through 2 years. The dotted line indicates the ODI score necessary for minimum clinical improvement, 10 points. ${ }^{14}$ (a) Mean scores for 276 subjects in the Charite artificial disc treatment group. There was a significant difference in level of disability at all time points compared to baseline $(P<.001)$, Wilcoxon rank sum test. (b) Mean scores for subjects in the treatment group compared to 99 enrolled subjects in the control group. There was a significant difference in the treatment group with respect to disability, compared to the control group at all postoperative time points $(P<$ .05), Wilcoxon rank sum test. 
Figure 3
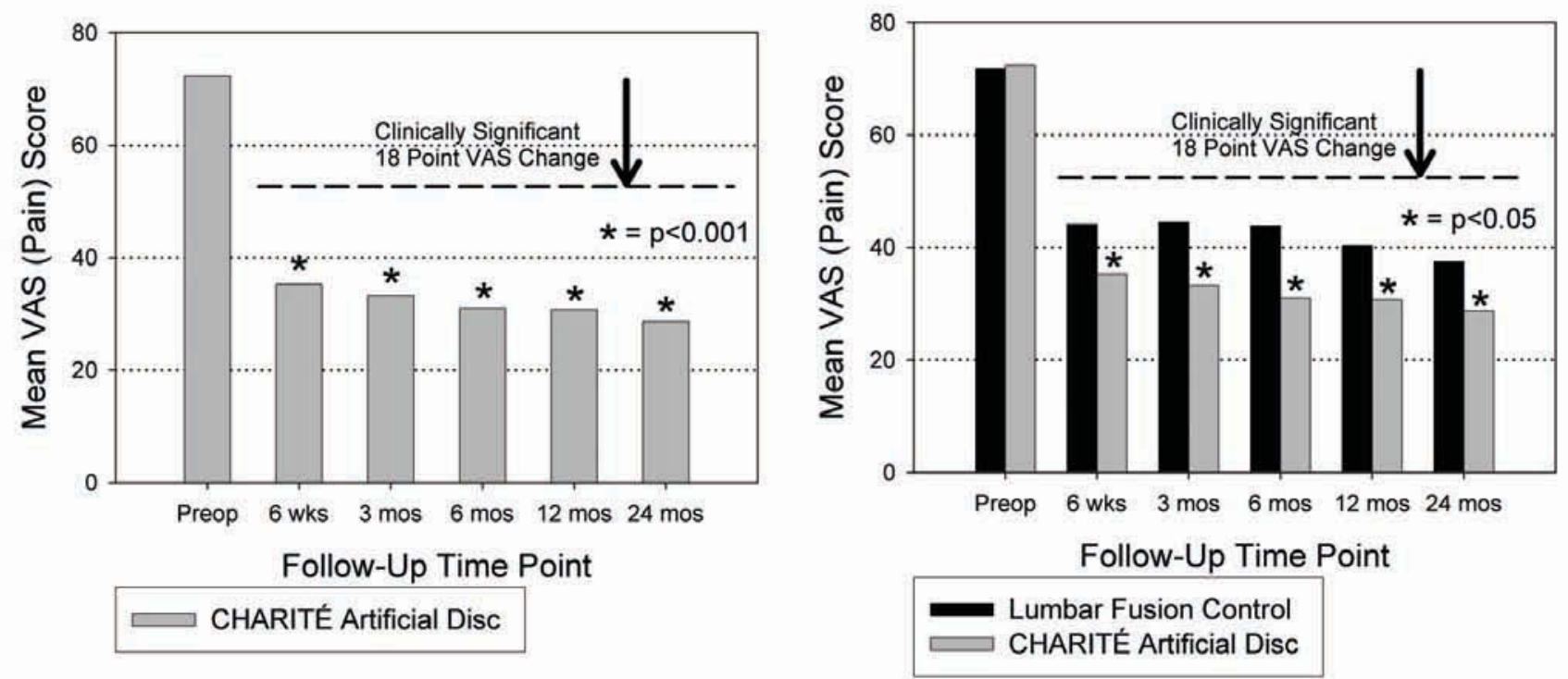

\begin{abstract}
Mean VAS pain scores at baseline and at each protocol-specified follow-up time point through 2 years. The dotted line indicates the VAS score necessary for minimum clinical improvement, 19 points. ${ }^{14}$ (a) Mean scores for 276 subjects in the Charite artificial disc treatment group. There was a significant difference in pain at all time points compared to baseline $(P<.001)$, Wilcoxon rank sum test. (b) Mean scores for subjects in the treatment group compared to the 99 enrolled subjects in the control group. There was a significant difference in the treatment group with respect to pain, compared to the control group at all postoperative points $(P<.05)$, Wilcoxon rank sum test.
\end{abstract}

all follow-up time points and attained superiority in pain and disability levels compared to fusion, the historical standard of care when the study began.

Deyo et al. have pointed out in a number of publications that, in their view, many treatments for low-back pain are ineffective, including osteopathic manipulation, ${ }^{17}$ chiropractic care, ${ }^{18}$ physical therapy,18 transcutaneous electrical nerve stimulation therapy, ${ }^{19}$ and fusion. ${ }^{20,21}$ Critics of lumbar arthroplasty cite mixed short-term ${ }^{22-25}$ and long-term ${ }^{26}$ results with the Charité artificial disc as well as review articles ${ }^{27,28}$ written before publication of the US trial (level IV medical evidence) as the primary reason why arthroplasty is not a reasonable treatment for discogenic low-back pain. Yet other more favorable long-term results in large patient cohorts (level IV medical evidence) are often only offhandedly considered. ${ }^{29-32}$

Lumbar arthroplasty with the Charité artificial disc has been performed outside the United States for more than 20 years and, as $\mathrm{McAfee}^{33}$ eloquently pointed out, the overwhelming majority of the early disc arthroplasty cases were performed with widely variable indications; basic, rudimentary instrumentation; different sizing options; nonexistent diagnostic testing; and a lack of fundamental understanding of lumbar spine biomechanics. These early issues and failures were well known to the clinical trial investigators at the time the FDA IDE study protocol was developed. In fact, it was this knowledge of the previous successes and failures that led to the inclusion/exclusion criteria and the surgical technique used in the FDA IDE study. Thus, the FDA IDE study was designed, based upon the earlier experience, to provide level I medical evidence of the efficacy and safety of treatment with lumbar arthroplasty learned from the earlier experience, and to better define the appropriate patient selection, surgical technique, and implant sizing. The previous lumbar artificial disc clinical information has been analyzed and clinical and surgical techniques have been refined through the 20-year history of the device. If this historical review of the previous series had not occurred, then the clinically superior results presented here would not have been possible. Using the historical data and clinical experience to criticize the FDA IDE results in a vacuum, without considering the advancements described above, seems unwarranted and unscientific.

As for lack of safety, another continuing cause for criticism of arthroplasty, the same historical literature is often cited without proper context and without any relevance to today's indications, implants, instrumentation, and knowledge. Contemporary information about complications in today's arthroplasty patients has been presented at dozens of medical meetings over the past 3 years and has been published. ${ }^{12,34-36}$ 
Revision rates for fusion are just as high or higher than for disc replacement. ${ }^{36,37}$ No evidence exists that the incidence of complications with or without revision is higher in arthroplasty patients compared with fusion patients. However, differences exist in the types of potential complications.

Before the 1970s-1980s, fusion was the standard of care for surgical treatment of degenerative conditions of the hip and knee. This fusion standard was replaced in the ensuing years with artificial joint arthroplasty, which today is the standard of care for surgical treatment of these conditions in indicated patients. Often cited as the grandfather of modern hip arthroplasty, the early ideas of John Charnley for avoiding fusion of the hip ${ }^{38-40}$ were not readily accepted. ${ }^{41,42}$ Though Charnley's work began in the late 1950s, the first hip replacement in the United States was not performed until 1969, with the first appearance in the US literature in $1970 .{ }^{43}$ Modern disc replacement has taken a similar track, with the third-generation Charité artificial disc having been developed and used in Europe as early as $1987^{44}$ and used in the first such procedure performed in the United States only in March 2000.

As noted earlier, Deyo et al. have denounced both non-operative and operative treatment for low-back pain, including fusion and now disc replacement. What else is there for the patient with chronic low-back pain? Despite Deyo's criticism, there are in fact multiple prospective, multicenter low-back fusion studies that describe good results and that have been published in the peerreviewed literature. ${ }^{16,45-47}$ All of these studies were performed under FDA-approved protocols with narrow indications, as was the Charité clinical trial.

Highly significant improvements in pain and level of disability compared to baseline in indicated disc replacement patients is not in question. Superior pain reduction and reduced disability level compared to the current standard of care are not in question. Critics of surgical intervention for patients with low-back pain decry the lack of level I data, yet when level I data demonstrating superior outcomes are produced, the surgical intervention is still criticized as "too new," "ineffective," or "unsafe" despite extraordinary evidence to the contrary. In the face of level I data, such critics use lower levels of evidence in an attempt to essentially cancel out the results of a level I study. But level II, III, $\mathrm{IV}$, and V data, by definition, do not trump level I data, and lower levels of published data exist for every treatment in medicine. If lower levels of data are allowed to trump Level I data, then nothing in medicine would be proven safe and effective, and if that is the case, why perform Level I studies at all?

Disc replacement in the low back is not for every patient with chronic low-back pain. Currently in the United States, disc replacement is narrowly indicated to patients with painful DDD at 1 level (L4-5 or L5-S1); who have no contraindications such as multilevel disease, scoliosis, or instability; and who fail at least 6 months of non-operative treatment. In my practice area, approximately 1 in 5000 patients with low-back pain are indicated for disc replacement after applying the indications and contraindications for the procedure. Thus, disc replacement is not being "sold" by physicians or by industry as a cure for lowback pain, though the lay press and Wall Street investors often jump to that conclusion.

As of this writing, a majority of private payers are not covering lumbar arthroplasty, a treatment backed by level I clinical data that demonstrates superior pain and disability improvement compared to baseline and to a fusion procedure. This leaves the patient with a choice between having an inferior surgical procedure covered by insurance or waiting in pain and disability for an indefinite period, hoping for a reversal in a payer's coverage decision. All of this takes the decision of treatment out of the hands of physicians and patients and places it in the capricious hands of government and third-party payers. As a result, only a small number of financially well-off patients will be able to receive this FDA-approved treatment. But the middle classand more importantly the working and nonworking poor-will not have access to an FDA-approved treatment in the United States. Further, though a CPT (current procedural terminology) code (22857) now exists for the procedure, the payment (\$1382) is decidedly inadequate compared with that for a less technically demanding ALIF procedure (\$1975), a disparity that serves as a disincentive to surgeons to perform lumbar arthroplasty in indicated patients.

Fred H. Geisler, MD, PhD

From the Illinois Neuro-Spine Center at Rush-Copley Medical Center, Aurora.

The author acknowledges consulting and research relationships with the manufacturer of the Charite artificial disc (DePuy Spine, Raynham, Mass), and has received funding in excess of $\$ 500$.

Address correspondence and reprint requests to Fred H. Geisler, $\mathrm{MD}, \mathrm{PhD}$, Illinois Neuro-Spine Center at Rush-Copley Medical Center, 2020 Ogden Ave, Suite 335, Aurora, IL 60504 (e-mail: fgeisler@concentric.net)

This submission was received December 7, 2006, and accepted for publication February 26, 2007.

Presented at the 6th Annual Meeting of the Spine Arthroplasty Society, May 9-13, 2006, in Montreal, Québec.

Institutional review board approval was obtained at the 14 hospital sites that participated in the original IDE study, in accordance with FDA regulations. 


\section{REFERENCES}

1. White AA, 3rd, Gordon SL. Synopsis: workshop on idiopathic low-back pain. Spine. 1982;7:141-149.

2. Bertagnoli R, Kumar S. Indications for full prosthetic disc arthroplasty: a correlation of clinical outcome against a variety of indications. Eur Spine J. 2002;11 Suppl 2:S131-S136.

3. Errico TJ. Lumbar disc arthroplasty. Clin Orthop Relat Res. 2005:106-117.

4. Davis TT, Delamarter RB, Sra P, et al. The IDET procedure for chronic discogenic low back pain. Spine. 2004;29:752-756.

5. Healy JF, Healy BB, Wong WH, et al. Cervical and lumbar MRI in asymptomatic older male lifelong athletes: frequency of degenerative findings. J Comput Assist Tomogr. 1996;20:107112.

6. Frymoyer JW. Back pain and sciatica. $N$ Engl $J$ Med. 1988;318:291-300.

7. Zitting P, Rantakallio P, Vanharanta H. Cumulative incidence of lumbar disc diseases leading to hospitalization up to the age of 28 years. Spine. 1998;23:2337-2343; discussion 43-4.

8. KA Martinelli, DT Lemaitre, TJ Lee. Orthopedic industry: Update and company models. Merrill Lynch, New York, 2000.

9. Geisler FH, Blumenthal SL, Guyer RD, et al. Neurological complications of lumbar artificial disc replacement and comparison of clinical results with those related to lumbar arthrodesis in the literature: results of a multicenter, prospective, randomized investigational device exemption study of Charité intervertebral disc. Invited submission from the Joint Section Meeting on Disorders of the Spine and Peripheral Nerves, March 2004. J Neurosurg Spine. 2004;1:143-154.

10. US Food and Drug Administration. CHARITÉ Artificial Disc summary of safety and effectiveness. PMA number P040006. October 26, 2004. 2004. Available at: http://www.fda.gov/cdrh/ PDF4/p040006.html. Accessed March 9, 2007.

11. Federal Register. Panel Transcript: Orthopedic and Rehabilitation Devices Panel of the Medical Devices Advisory Committee, 2004. Available at: http://www.fda.gov/ohrms/dockets/ac/04/ transcripts/2004-4049t1.htm. Accessed March 9, 2007.

12. Blumenthal SL, McAfee PC, Guyer RD, et al. A prospective, randomized, multicenter Food and Drug Administration investigational device exemption study of lumbar total disc replacement with the Charité artificial disc versus lumbar fusion part I: Evaluation of clinical outcomes. Spine. 2005;30:1565-1575.
13. McAfee PC, Cunningham B, Holsapple GA, et al. A prospective, randomized, multicenter Food and Drug Administration investigational device exemption study of lumbar total disc replacement with the Charité artificial disc versus lumbar fusion part II: Evaluation of radiographic outcomes and correlation of surgical technique accuracy with clinical outcomes. Spine. 2005;30:1576-1583.

14. Fairbank JC, Pynsent PB. The Oswestry Disability Index. Spine. 2000;25:2940-2952.

15. Hagg O, Fritzell P, Ekselius L, et al. Predictors of outcome in fusion surgery for chronic low back pain. A report from the Swedish Lumbar Spine Study. Eur Spine J. 2003;12:22-33.

16. Kuslich SD, Ulstrom CL, Griffith SL, et al. The Bagby and Kuslich method of lumbar interbody fusion. History, techniques, and 2-year follow-up results of a United States prospective, multicenter trial. Spine. 1998;23:1267-1278; discussion 79.

17. Andersson GB, Lucente T, Davis AM, et al. A comparison of osteopathic spinal manipulation with standard care for patients with low back pain. $N$ Engl J Med. 1999;341:1426-1431.

18. Cherkin DC, Deyo RA, Battie M, et al. A comparison of physical therapy, chiropractic manipulation, and provision of an educational booklet for the treatment of patients with low back pain. N Engl J Med. 1998;339:1021-1029.

19. Deyo RA, Walsh NE, Martin DC, et al. A controlled trial of transcutaneous electrical nerve stimulation (TENS) and exercise for chronic low back pain. N Engl J Med. 1990;322:1627-1634.

20. Deyo RA, Nachemson A, Mirza SK. Spinal-fusion surgery - the case for restraint. $N$ Engl J Med. 2004;350:722-726.

21. Deyo RA, Weinstein JN. Low back pain. $N$ Engl J Med. 2001;344:363-370.

22. Cinotti G, David T, Postacchini F. Results of disc prosthesis after a minimum follow-up period of 2 years. Spine. 1996;21:995-1000.

23. Zeegers WS, Bohnen LM, Laaper M, et al. Artificial disc replacement with the modular type SB Charité III: 2-year results in 50 prospectively studied patients. Eur Spine J. 1999;8:210-217.

24. van Ooij A, Oner FC, Verbout AJ. Complications of artificial disc replacement: a report of 27 patients with the SB Charité disc. $J$ Spinal Disord Tech. 2003;16:369-383.

25. Griffith SL, Shelokov AP, Büttner-Janz K, et al. A multicenter retrospective study of the clinical results of the LINK SB Charite intervertebral prosthesis. The initial European experience. Spine. 1994;19:1842-1849. 
26. Putzier M, Funk JF, Schneider SV, et al. Charité total disc replacementclinical and radiographical results after an average follow-up of 17 years. Eur Spine J. 2005: published online October 28, 2005.

27. Gamradt SC, Wang JC. Lumbar disc arthroplasty. Spine J. 2005;5:95103.

28. de Kleuver M, Oner FC, Jacobs WC. Total disc replacement for chronic low back pain: background and a systematic review of the literature. Eur Spine J. 2003;12:108-116.

29. Lemaire JP, Skalli W, Lavaste F, et al. Intervertebral disc prosthesis. Results and prospects for the year 2000. Clin Orthop. 1997:64-76.

30. Lemaire JP, Carrier H, Sari Ali E, et al. Clinical and radiological outcomes with the Charite artificial disc: A 10-year minimum followup. J Spinal Disord. 2005;18:353-359.

31. David T. Lumbar disc prosthesis: Five years' follow-up study on 96 patients. Presented at the 15th Annual Meeting of the North American Spine Society. New Orleans, Louisiana, 2000.

32. David T. Lumbar disc prosthesis: An analysis of long-term complications for 272 Charite artificial disc prostheses with minimum 10-year follow-up. Spine J. 2004;4:S50-S51.

33. McAfee PC. Comments on the van Ooij article. J Spinal Disord Tech. 2005;18:116-117.

34. David T. Revision of a Charité artificial disc 9.5 years in vivo to a new Charité artificial disc: case report and explant analysis. Eur Spine J. 2005;14:507-511.

35. Regan JJ, McAfee PC, Blumenthal SL, et al. Evaluation of surgical volume and the early experience with lumbar total disc replacement as part of the investigational device exemption study of the Charite artificial disc. Spine. 2006;31:2270-2276.

36. McAfee PC, Geisler FH, Saiedy S, et al. Revisability of the Charite artificial disc replacement-analysis of 688 patients enrolled in the US IDE study of the Charité artificial disc. Spine. 2006;31:1217-1226.

37. Blumenthal SL, McAfee PC, Geisler FH, et al. Response to the editorial and points of view regarding the IDE study of the Charité artificial disc. Spine. 2005;30:Linked via Article Plus to Zindrick et al E388-390. Available at: http://acs.ovid.com/acs/

38. Charnley J, Wright JK. A spring exerciser for arthroplasty of the hip joint. J Bone Joint Surg Br. 1951;33-B:634-635.

39. Charnley J. Surgery of the hip-joint: present and future developments. Br Med J. 1960;5176:821-826.

40. Charnley J. Arthroplasty of the hip. A new operation. Lancet. 1961;1:1129-1132.
41. Chapchal G. [Critique of arthroplasty of the hip joint with special reference to further therapy following defective results.]. Ann Univ Sarav [Med]. 1958;6:280-281.

42. Chapchal G. [Critical remarks on arthroplasty of the hip with reference to further treatment in poor results.]. Medizinische. 1958;12:560-564.

43. Nelson JP, Talbott RD, Glassburn AR Jr. Charnley total hip arthroplasty: A preliminary report. Rocky Mt Med J. 1970;67:2526.

44. Link HD. History, design, and biomechanics of the LINK SB Charité artificial disc. Eur Spine J. 2002;11 Suppl 2:S98-S105.

45. Brantigan JW, Steffee AD, Lewis ML, et al. Lumbar interbody fusion using the Brantigan $\mathrm{I} / \mathrm{F}$ cage for posterior lumbar interbody fusion and the variable pedicle screw placement system: Twoyear results from a Food and Drug Administration investigational device exemption clinical trial. Spine. 2000;25:1437-1446.

46. Burkus JK, Gornet MF, Dickman CA, et al. Anterior lumbar interbody fusion using rhBMP-2 with tapered interbody cages. $J$ Spinal Disord Tech. 2002;15:337-349.

47. Ray CD. Threaded titanium cages for lumbar interbody fusions. Spine. 1997;22:667-679; discussion 679-680.

48. bb350cc260751a2bb065792ca35d33103c0cc2f8a1b74b419f80e 8c57b0ee4bfbe2eb74552cd48915ce951016e6950fa7611d78f20d 752c0387c3de10d7b35de.pdf. Accessed March 9, 2007. 